Supporting information for

\title{
ReaxFF Reactive Molecular Dynamics Simulation of Functionalized Polyphenylene Oxide Anion Exchange Membrane
}

\author{
Weiwei Zhang, Adri C. T. van Duin*
}

Department of Mechanical and Nuclear Engineering,

Pennsylvania State University, University Park, Pennsylvania

$$
\text { 16802, USA }
$$

E-mail: acv13@psu.edu

CONTENTS:

1. ReaxFF reactive force field parameters for hydrated membrane.

2. Complete citation for reference 33 . 


\section{Reactive MD-force field:}

39 ! Number of general parameters

50.0000 !Overcoordination parameter

9.5469 !Overcoordination parameter

1.6725 !Valency angle conjugation parameter

1.7224 !Triple bond stabilisation parameter

6.8702 !Triple bond stabilisation parameter

60.4850 !C2-correction

1.0588 !Undercoordination parameter

4.6000 !Triple bond stabilisation parameter

12.1176 !Undercoordination parameter

13.3056 !Undercoordination parameter

-40.0000 !Triple bond stabilization energy

0.0000 !Lower Taper-radius

10.0000 !Upper Taper-radius

2.8793 !Not used

33.8667!Valency undercoordination

6.0891 !Valency angle/lone pair parameter

1.0563 !Valency angle

2.0384 !Valency angle parameter

6.1431 !Not used

6.9290 !Double bond/angle parameter

0.3989 !Double bond/angle parameter: overcoord

3.9954 !Double bond/angle parameter: overcoord

-2.4837 !Not used

5.7796 !Torsion/BO parameter

10.0000 !Torsion overcoordination

1.9487 !Torsion overcoordination

-1.2327 !Conjugation 0 (not used)

2.1645 !Conjugation

1.5591 !vdWaals shielding

0.1000 !Cutoff for bond order $(* 100)$

1.7602 !Valency angle conjugation parameter

0.6991 !Overcoordination parameter

50.0000 !Overcoordination parameter

1.8512 !Valency/lone pair parameter

0.5000 !Not used

20.0000 !Not used

5.0000 !Molecular energy (not used)

0.0000 !Molecular energy (not used)

0.7903 !Valency angle conjugation parameter

4 ! Nr of atoms; cov.r; valency;a.m;Rvdw;Evdw;gammaEEM;cov.r2;\# alfa;gammavdW;valency;Eunder;Eover;chiEEM;etaEEM;n.u. cov r3;Elp;Heat inc.;n.u.;n.u.;n.u.;n.u. 


\begin{tabular}{ccccccccc}
\multicolumn{7}{c}{ ov/un;val1;n.u.;val3,vval4 } & & \\
$\mathrm{C}$ & 1.3817 & 4.0000 & 12.0000 & 1.8903 & 0.1838 & 0.6621 & 1.1341 & 4.0000 \\
& 9.7559 & 2.1346 & 4.0000 & 34.9350 & 79.5548 & 5.1015 & 6.0000 & 0.0000 \\
& 1.2114 & 0.0000 & 202.2908 & 8.9539 & 34.9289 & 13.5366 & 0.8563 & 0.0000 \\
& -2.8983 & 2.5000 & 1.0564 & 4.0000 & 2.9663 & 0.0000 & 0.0000 & 0.0000 \\
$\mathrm{H}$ & 0.8930 & 1.0000 & 1.0080 & 1.3550 & 0.0930 & 0.8203 & -0.1000 & 1.0000 \\
& 8.2230 & 33.2894 & 1.0000 & 0.0000 & 121.1250 & 3.7248 & 9.6093 & 1.0000 \\
& -0.1000 & 0.0000 & 55.1878 & 3.0408 & 2.4197 & 0.0003 & 1.0698 & 0.0000 \\
& -19.4571 & 4.2733 & 1.0338 & 1.0000 & 2.8793 & 0.0000 & 0.0000 & 0.0000 \\
$\mathrm{O}$ & 1.2450 & 2.0000 & 15.9990 & 2.3890 & 0.1000 & 1.0898 & 1.0548 & 6.0000 \\
& 9.7300 & 13.8449 & 4.0000 & 37.5000 & 116.0768 & 8.5000 & 8.3122 & 2.0000 \\
& 0.9049 & 0.4056 & 68.0152 & 3.5027 & 0.7640 & 0.0021 & 0.9745 & 0.0000 \\
& -3.5500 & 2.9000 & 1.0493 & 4.0000 & 2.9225 & 0.0000 & 0.0000 & 0.0000 \\
$\mathrm{~N}$ & 1.2333 & 3.0000 & 14.0000 & 2.1132 & 0.1167 & 1.0000 & 1.1748 & 5.0000 \\
& 10.1084 & 9.4128 & 4.0000 & 32.5000 & 100.0000 & 6.5896 & 7.0387 & 2.0000 \\
& 1.0433 & 2.6737 & 119.9837 & 0.5817 & 8.3001 & 2.2372 & 0.9745 & 0.0000 \\
& -4.4463 & 4.0000 & 1.0183 & 4.0000 & 2.8793 & 0.0000 & 0.0000 & 0.0000
\end{tabular}

10 ! Nr of bonds; Edis1;LPpen;n.u.;pbe1;pbo5;13corr;pbo6

pbe2;pbo3;pbo4;Etrip;pbo1;pbo2;ovcorr

\begin{tabular}{|c|c|c|c|c|c|c|c|c|}
\hline & & & & & & & & \\
\hline & 0.4590 & - & & & -0 & & & \\
\hline & 50 & & & -( & & & & \\
\hline & 5.2 & 00 & 00 & 00 & & & & 000 \\
\hline & 3.39 & 0.0 & & -0.4 & & & & \\
\hline & & & & & & & & \\
\hline & 3121.66581 & 0038 & 3. & 0.2759 & -0.3 & 00 & 78 & 0.9686 \\
\hline & & & & & & & & \\
\hline & 42.2 & 145. & 0 & 0.2 & & & 29.7 & \\
\hline & & & & & & & & \\
\hline & 4 & 138.3 & .1177 & .8108 & -0.6 & 1.0000 & 7.62 & 0.20 \\
\hline & 96 & -0. & 30 & 1.0000 & & 25 & 00 & 00 \\
\hline & 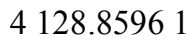 & & 40.0000 & & -0 . & 00 & & \\
\hline & & & & & & & & \\
\hline & 4160.1592 & 82.55261 & 384 & 0.4110 & -0.0 & 1.0000 & 12.4304 & 0.5899 \\
\hline & & & & 0000 & & & 00 & 000 \\
\hline & (2) & & & 5725 & & & & \\
\hline & & & & 0 & 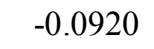 & & & \\
\hline & כסוס.Jנ & & & 30 & & & 000 & 0.5203 \\
\hline & 2.7086 & 1.0000 & 0.0000 & 1.0000 & -0.1598 & 5.7729 & 0.0000 & 0.0000 \\
\hline
\end{tabular}

6 ! Nr of off-diagonal terms; Ediss;Ro;gamma;rsigma;rpi;rpi2

$\begin{array}{rrrrrrrr}1 & 2 & 0.1239 & 1.4004 & 9.8467 & 1.1210 & -1.0000 & -1.0000 \\ 2 & 3 & 0.0283 & 1.2885 & 10.9190 & 0.9215 & -1.0000 & -1.0000 \\ 2 & 4 & 0.1751 & 1.3144 & 9.5031 & 1.0557 & -1.0000 & -1.0000 \\ 1 & 3 & 0.0753 & 2.0418 & 9.9374 & 1.3613 & 1.1866 & 1.0722\end{array}$




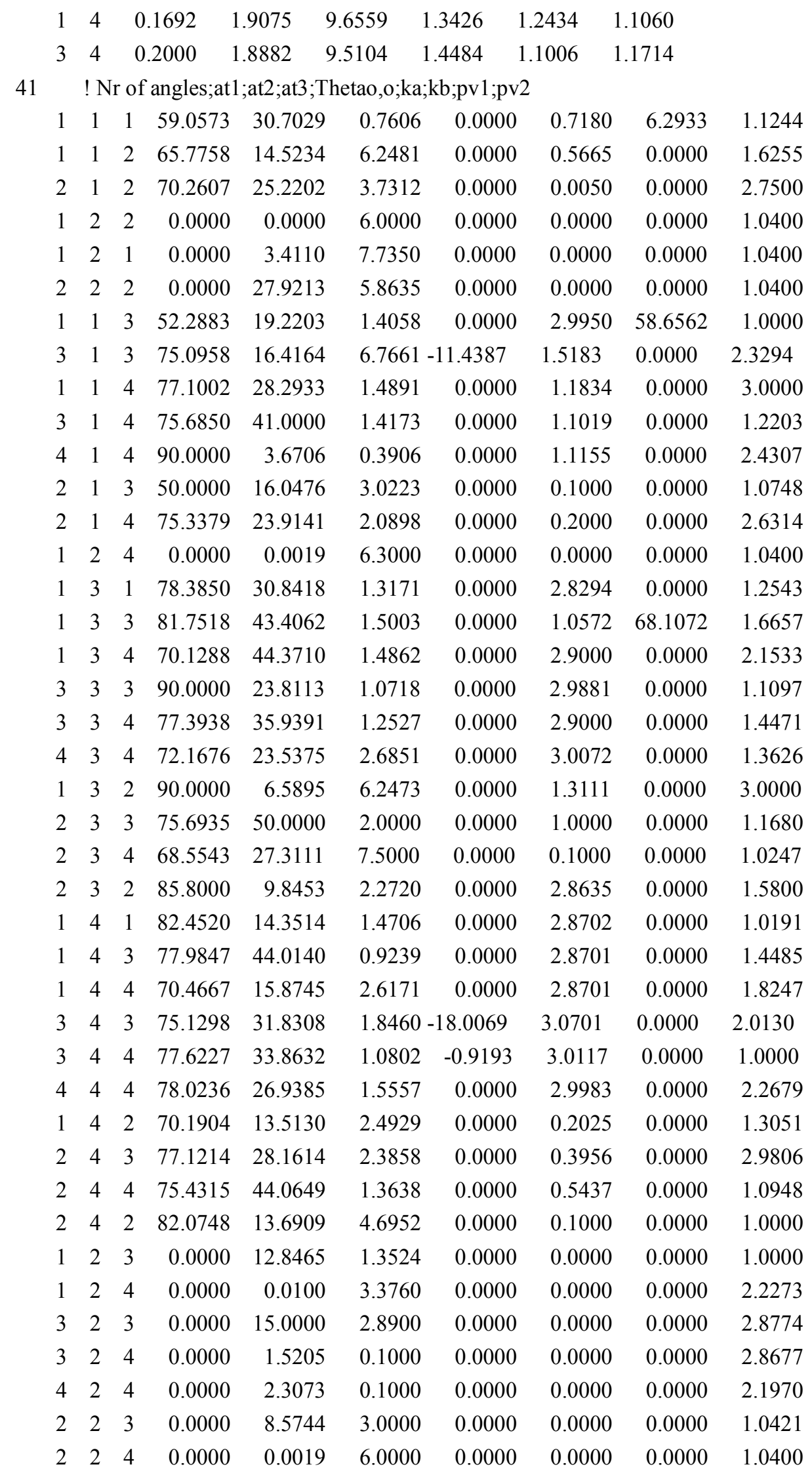


$41 \quad$ ! Nr of torsions;at1;at2;at3;at4;:V1;V2;V3;V2(BO);vconj;n.u;n

\begin{tabular}{|c|c|c|c|c|c|c|c|c|c|c|}
\hline & 1 & 1 & 1 & -0.2500 & 34.7453 & 0.0288 & -6.3507 & -1.6000 & 0.0000 & 0.0000 \\
\hline & 1 & 1 & 2 & -0.2500 & 29.2131 & 0.2945 & -4.9581 & -2.1802 & 0.0000 & 0.0000 \\
\hline & 1 & 1 & 2 & -0.2500 & 31.2081 & 0.4539 & -4.8923 & -2.2677 & 0.0000 & 0.0000 \\
\hline & 1 & 1 & 3 & -2.5000 & 73.9456 & 1.0000 & -4.9664 & -1.1000 & 0.0000 & 0.0000 \\
\hline & 1 & 1 & 3 & -0.2297 & 46.7166 & 0.9972 & -6.3813 & -1.0978 & 0.0000 & 0.0000 \\
\hline & 1 & 1 & 3 & -2.2371 & 16.9063 & -0.5851 & -3.7433 & -0.8614 & 0.0000 & 0.0000 \\
\hline & 1 & 3 & 1 & -2.5000 & 80.0000 & -1.0000 & -6.4787 & -0.9000 & 0.0000 & 0.0000 \\
\hline & 1 & 3 & 2 & 2.5000 & 14.6769 & -1.0000 & -3.7197 & -0.9000 & 0.0000 & 0.0000 \\
\hline & 1 & 3 & 1 & -2.5000 & 80.0000 & 0.4547 & -6.1301 & -0.9000 & 0.0000 & 0.0000 \\
\hline & 1 & 3 & 2 & -1.0000 & 33.9036 & 0.9140 & -2.5069 & -1.1000 & 0.0000 & 0.0000 \\
\hline & 1 & 3 & 3 & 2.5000 & 80.0000 & 1.0000 & -2.5000 & -2.8274 & 0.0000 & .0000 \\
\hline & 1 & 3 & 1 & -1.7428 & 77.3763 & -0.3420 & -4.1374 & -3.0437 & 0.0000 & 0.0000 \\
\hline & 1 & 3 & 2 & 2.4786 & 34.1116 & 0.7529 & -2.5000 & -3.0476 & 0.0000 & 0.0000 \\
\hline & 3 & 3 & 2 & -1.0000 & -5.0000 & 0.4774 & -2.7138 & -2.9498 & 0.0000 & 0.0000 \\
\hline & 3 & 3 & 2 & 2.5000 & 5.0000 & -1.0000 & -4.4389 & 0.0000 & 0.0000 & 0.0000 \\
\hline & 3 & 3 & 3 & $2.1682-$ & -13.6133 & 0.9044 & -2.5783 & -0.9972 & 0.0000 & 0.0000 \\
\hline & 3 & 3 & 3 & -2.5000 & 62.3765 & -1.0000 & -7.9091 & 0.0000 & 0.0000 & 0.0000 \\
\hline & 3 & 3 & 3 & -2.5000 & 25.0000 & 1.0000 & -2.5000 & -0.9 & 0.0000 & 0.0000 \\
\hline & 1 & 4 & 2 & -0.0647 & 63.1787 & -0.0726 & -9.0000 & -1.9825 & 0.0000 & 0.0000 \\
\hline & 1 & 4 & 2 & -1.7861 & 70.4826 & 0.3956 & 86 & -2 . & 00 & 000 \\
\hline & 1 & 4 & 2 & 1.3753 & 44.5729 & 1.0000 & -4.9670 & -2.5261 & 0.0000 & 0.0000 \\
\hline & 1 & 1 & 4 & -0.23391 & 00.0000 & 0.8363 & -5.0470 & -0.9511 & 0.0000 & 0.0000 \\
\hline & 1 & 1 & 4 & 0.7316 & 100.0000 & 0.8200 & -3.7644 & -1.7241 & 0.0000 & 0.0000 \\
\hline & 1 & 4 & 1 & 0.3377 & 7.2105 & 0.8382 & -3.3034 & -1.6589 & 0.0000 & 0.0000 \\
\hline & 1 & 4 & 1 & -1.0000 & -3.8255 & 0.6171 & -5.5850 & -1.8038 & 0.0000 & 0.0000 \\
\hline & 1 & 1 & 4 & 1.0000 & 16.8673 & 0.2359 & -2.5000 & -1.9000 & 0.0000 & 0.0000 \\
\hline & 1 & 4 & 2 & 1.00001 & 100.0000 & 1.0000 & -5.9551 & -2.0202 & 0.0000 & 0.0000 \\
\hline & 1 & 4 & 1 & -1.0000 & 51.8333 & -0.4257 & -7.8922 & -1.5996 & 0.0000 & 0.0000 \\
\hline & 1 & 2 & 0 & -1.00001 & 00.0000 & -0.5000 & -3.7543 & 0.0000 & 0.0000 & 0.0000 \\
\hline & 2 & 2 & 0 & 1.00001 & 100.0000 & -0.3126 & -2.5000 & 0.0000 & 0.0000 & 0.0000 \\
\hline & 2 & 3 & 0 & -1.0000 & 35.6414 & -0.5000 & -2.8324 & 0.0000 & 0.0000 & 0.0000 \\
\hline & 1 & 1 & 0 & 0.0000 & 50.0000 & 0.3000 & -4.0000 & -2.0000 & 0.0000 & 0.0000 \\
\hline & 3 & 3 & 0 & 0.5511 & 25.4150 & 1.1330 & -5.1903 & -1.0000 & 0.0000 & 0.0000 \\
\hline & 1 & 4 & 0 & 0.2176 & 40.4126 & 0.3535 & -3.9875 & -2.0051 & 0.0000 & 0.0000 \\
\hline & 2 & 4 & 0 & 0.0000 & 0.1032 & 0.3000 & -5.0965 & 0.0000 & 0.0000 & 0.0000 \\
\hline & 3 & 4 & 0 & 1.1397 & 61.3225 & 0.5139 & -3.8507 & -2.7831 & 0.0000 & 0.0000 \\
\hline & 4 & 4 & 0 & 0.7265 & 44.3155 & 1.0000 & -4.4046 & -2.0000 & 0.0000 & 0.0000 \\
\hline & 1 & 4 & 4 & -0.0949 & 8.7582 & 0.3310 & -7.9430 & -2.0000 & 0.0000 & 0.0000 \\
\hline & 1 & 3 & 3 & -0.0002 & 20.1851 & 0.1601 & -9.0000 & -2.0000 & 0.0000 & 0.0000 \\
\hline & 3 & 3 & 1 & 0.0002 & 80.0000 & -1.5000 & -4.4848 & -2.0000 & 0.0000 & 0.0000 \\
\hline & 1 & 3 & 3 & -0.1583 & 20.0000 & 1.5000 & -9.0000 & -2.0000 & 0.0000 & 0.0000 \\
\hline \multicolumn{11}{|c|}{ Jr of hydrogen bonds;at1;at2;at3;Rhb;Dehb;vhb1 } \\
\hline & & 3 & 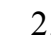 & 200 & 300 & 00 & 000 & & & \\
\hline
\end{tabular}




$\begin{array}{lllllll}3 & 2 & 4 & 2.0858 & -4.5000 & 1.4500 & 19.5000 \\ 4 & 2 & 3 & 1.7500 & -1.5172 & 1.4500 & 19.5000 \\ 4 & 2 & 4 & 1.9274 & -1.6801 & 1.4500 & 19.5000\end{array}$

\section{Reference 33}

Achtyl J. L.; Unocic R. R.; Xu L.; Cai Y.; Raju M.; Zhang W.; Sacci R. L.; Vlassiouk I. V.; Fulvio P. F.; Ganesh P.; Wesolowski D. J.; Dai S.; van Duin A. C. T.; Neurock, M.; Geiger F. M., Aqueous Proton Transfer Across Single-Layer Graphene. Nat. Commun. 2015, 6, 6539 . 